\title{
Glycerol: An unexpected major metabolite of energy metabolism by the human malaria parasite
}

\author{
Lu-Yun Lian'1, Mohammed Al-Helal², Abd Majid Roslaini², Nicholas Fisher ${ }^{2}$, \\ Patrick G Bray ${ }^{2}$, Stephen A Ward ${ }^{2}$ and Giancarlo A Biagini*2
}

\author{
Address: ${ }^{1}$ School of Biological Sciences, Biosciences Building, University of Liverpool, P.O. Box 147, Liverpool, L69 7ZB, UK and ${ }^{2}$ Liverpool School \\ of Tropical Medicine, Liverpool, L35 QA, UK \\ Email: Lu-Yun Lian - lylian1@liv.ac.uk; Mohammed Al-Helal - alhelal@liv.ac.uk; Abd Majid Roslaini - doss1107@liv.ac.uk; \\ Nicholas Fisher - n.e.fisher@liv.ac.uk; Patrick G Bray - pgbray@liv.ac.uk; Stephen A Ward - saward@liv.ac.uk; \\ Giancarlo A Biagini* - Biagini@liv.ac.uk \\ * Corresponding author
}

Published: 6 March 2009

Malaria Journal 2009, 8:38 doi:10.1186/1475-2875-8-38

This article is available from: http://www.malariajournal.com/content/8/I/38

(C) 2009 Lian et al; licensee BioMed Central Ltd.

This is an Open Access article distributed under the terms of the Creative Commons Attribution License (http://creativecommons.org/licenses/by/2.0), which permits unrestricted use, distribution, and reproduction in any medium, provided the original work is properly cited.

\begin{abstract}
Background: Malaria is a global health emergency, and yet our understanding of the energy metabolism of the principle causative agent of this devastating disease, Plasmodium falciparum, remains rather basic. Glucose was shown to be an essential nutritional requirement nearly 100 years ago and since this original observation, much of the current knowledge of Plasmodium energy metabolism is based on early biochemical work, performed using basic analytical techniques (e.g. paper chromatography), carried out almost exclusively on avian and rodent malaria. Data derived from malaria parasite genome and transcriptome studies suggest that the energy metabolism of the parasite may be more complex than hitherto anticipated. This study was undertaken in order to further characterize the fate of glucose catabolism in the human malaria parasite, P. falciparum.
\end{abstract}

Methods: Products of glucose catabolism were determined by incubating erythrocyte-freed parasites with D-[I${ }^{13} \mathrm{C}$ ] glucose under controlled conditions and metabolites were identified using ${ }^{13} \mathrm{C}-\mathrm{NMR}$ spectroscopy.

Results: Following a $2 \mathrm{~h}$ incubation of freed-P. falciparum parasites with $25 \mathrm{mM} \mathrm{D}-\left[\mathrm{I}-{ }^{13} \mathrm{C}\right]$ glucose $(n=4)$, the major metabolites identified included; $\left[3-{ }^{13} \mathrm{C}\right]$ lactate, $\left[1,3-{ }^{13} \mathrm{C}\right]$ glycerol, $\left[3-{ }^{13} \mathrm{C}\right]$ pyruvate, $\left[3^{13} \mathrm{C}\right]$ alanine and $[3-$ $\left.{ }^{13} \mathrm{C}\right]$ glycerol-3-phosphate. Control experiments performed with uninfected erythrocytes incubated under identical conditions did not show any metabolism of $D-\left[1-{ }^{3} \mathrm{C}\right]$ glucose to glycerol or glycerol-3-phosphate.

Discussion: The identification of glycerol as a major glucose metabolite confirms the view that energy metabolism in this parasite is more complex than previously proposed. It is hypothesized here that glycerol production by the malaria parasite is the result of a metabolic adaptation to growth in $\mathrm{O}_{2}$-limited (and $\mathrm{CO}_{2}$ elevated) conditions by the operation of a glycerol-3-phosphate shuttle for the re-oxidation of assimilatory NADH. Similar metabolic adaptations have been reported previously for other microaerobic/anaerobic organisms, such as yeast, rumen protozoa and human parasitic protozoa.

Conclusion: These data highlight the need to re-evaluate the carbon and redox balance of this important human pathogen, ultimately leading to a better understanding of how the parasite is able to adapt to the variable environments encountered during parasite development and disease progression. 


\section{Background}

Despite the clinical and economic significance of the human malaria parasite, Plasmodium falciparum, the energy metabolism of this organism is still poorly understood. Glucose was shown to be an essential nutritional requirement nearly 100 years ago [1] and experiments performed using $\left[{ }^{14} \mathrm{C}\right]$-glucose revealed that lactate is the major detectable end product of glucose catabolism together with a variety of other organic acids (e.g. pyruvate, acetate, succinate, aspartate, glutamate, alanine and $\mathrm{CO}_{2}$ ) depending on the species of malaria [2]. It is perhaps surprising to note that much of this early biochemical work was performed almost exclusively on avian and rodent malaria and very little information exists regarding human malaria parasites.

It is clear from genome sequence information, that the energy metabolism of $P$. falciparum is more complex than hitherto anticipated and an almost full compliment of tricarboxylic acid (TCA) cycle and electron transport chain (ETC) genes have so far been identified [3-5]. Subsequent transcriptome data indicates an up-regulation of expression of many of these genes during the transition from asexual to sexual stages [6-8] and more recently there is evidence of different expression profiles of in vivo intraerythrocytic parasites compared to laboratory cultured parasites [9].

However, without biochemical data the role of each of these components and their interdependence remains hypothetical. This study has, therefore, set out to perform a qualitative analysis of glucose catabolism using ${ }^{13} \mathrm{C}$ NMR. The advantage of this technique is that it allows the identification of metabolites without the a priory need of information regarding the products. This study reports that glycerol is an unexpected product of glucose catabolism in the human malaria parasite P. falciparum, and the role played by this pathway in maintaining the redox balance of the cell is discussed.

\section{Methods}

\section{Parasite culture and free parasite preparation}

Plasmodium falciparum strain 3D7 was maintained in continuous culture in a $2 \%$ suspension of $\mathrm{O}+$ erythrocytes in RPMI 1640 medium (R8758, glutamine, and NaHCO3) supplemented with $10 \%$ pooled human serum, $25 \mathrm{mM}$ HEPES (pH 7.4), and $20 \mu \mathrm{M}$ gentamicin sulphate[10].

Preparation of free parasites, from an aliquot of infected erythrocytes, was performed by centrifugation $(3,000 \times \mathrm{g}$, $5 \mathrm{~min}$ ) and resuspension of the pellet in $5 \mathrm{vol}$ of $0.15 \%$ (wt/vol) saponin in phosphate-buffered saline (PBS) for 1 min, followed by three washes by centrifugation and resuspension in Ringers $\left(106 \mathrm{mM} \mathrm{NaCl}, 24 \mathrm{mM} \mathrm{NaHCO}_{3}\right.$ $5.4 \mathrm{mM} \mathrm{KCl}, 1.2 \mathrm{mM} \mathrm{CaCl}_{2}, 1 \mathrm{mM} \mathrm{Na} \mathrm{HPO}_{4}, 0.8 \mathrm{mM}$ $\mathrm{MgCl}_{2}, 5.5 \mathrm{mM}$ D-glucose, $\mathrm{pH} 7.4$ ) and two washes in glu- cose-free Ringers. Samples were checked microscopically to ensure lysis of all erythrocytes. It is worth noting that there is a large body of evidence to show that $P$. falciparum parasites isolated using the saponin lysis technique remain viable for at least $2 \mathrm{~h}$ as demonstrated by the maintenance of the parasite plasma membrane potential [11], maintenance of the ATP/ADP couple [12] and the operation of a number of $\mathrm{H}+-$ and $\mathrm{Na}+$-dependent transporters, which can only operate when the integrity of the plasma membrane electrochemical gradient is maintained e.g. [13-15].

\section{NMR spectroscopy measurements}

Products of glucose fermentation were identified by incubating erythrocyte freed-parasites at $37^{\circ} \mathrm{C}$ under $3 \% \mathrm{O}_{2} /$ $4 \% \mathrm{CO}_{2}$ in $\mathrm{N}_{2}$ for $2 \mathrm{~h}$, in Ringers ( $\mathrm{pH} 7.4$ ) containing 25 $\mathrm{mM}$ D-[1-13 C] glucose. Following incubation, cell suspensions were disrupted by probe sonication, centrifuged (13 $000 \mathrm{~g} \times 2 \mathrm{~min}$ ) and the supernatants collected for analyses. Proton-decoupled ${ }^{13} \mathrm{C}-\mathrm{NMR}$ spectra were recorded at $600 \mathrm{MHz}$ on a Bruker Avance spectrometer equipped with a triple resonance cryoprobe. Free induction decay was measured for a total of 26,000 data points covering a spectral width of $197 \mathrm{ppm}$ with pulses of $10.3 \mathrm{~ms}\left(90^{\circ}\right)$ at $2 \mathrm{~s}$ intervals. ${ }^{2} \mathrm{H}_{2} \mathrm{O}$ was used as the internal lock. Chemical shifts, in parts per million, were measured with respect to the $\mathrm{bC}-1$ resonance in the added D-glucose $(97.0 \mathrm{ppm})$ [16]. Data presented are representative of 4 independent experiments. Spectra were also recorded for control experiments performed under identical conditions with uninfected human erythrocytes (5\% suspension).

\section{Results and Discussion}

It has long been the held view that lactate is the sole endproduct of glucose fermentation by P. falciparum [2]. By following the catabolism of D-[1-13 C] glucose by ${ }^{13} \mathrm{C}$ NMR, this study has identified that in addition to lactate, glycerol (and glycerol phosphate), pyruvate and alanine are significant end products of glucose fermentation. A typical ${ }^{13} \mathrm{C}-\mathrm{NMR}$ spectrum of erythrocyte-freed trophozoite stage $P$. falciparum following $2 \mathrm{~h}$ incubation with 25 $\mathrm{mM}$ D- $\left[1-{ }^{13} \mathrm{C}\right]$ glucose is shown in Fig. 1. Metabolites were identified according to previously reported shifts (ppm)[16], these included $\left[3-{ }^{13} \mathrm{C}\right]$ lactate $(21.2 \mathrm{ppm})$, $\left[1,3-{ }^{13} \mathrm{C}\right]$ glycerol $(63.6 \mathrm{ppm}),\left[3-{ }^{13} \mathrm{C}\right]$ pyruvate $(27.6$ ppm), [3-13 C] alanine (17.3 ppm) and [3-13 C] glycerol-3phosphate (65.9 ppm) and also by spiking test samples with known reference compounds (performed for each experiment, $n=4)$. Control experiments performed with uninfected erythrocytes incubated under identical conditions did not show any metabolism of D-[1-13C] glucose to glycerol or glycerol-3-phosphate.

The identification of additional glucose catabolism endproducts raises several questions regarding our understanding of the carbon and redox balance of the malaria 


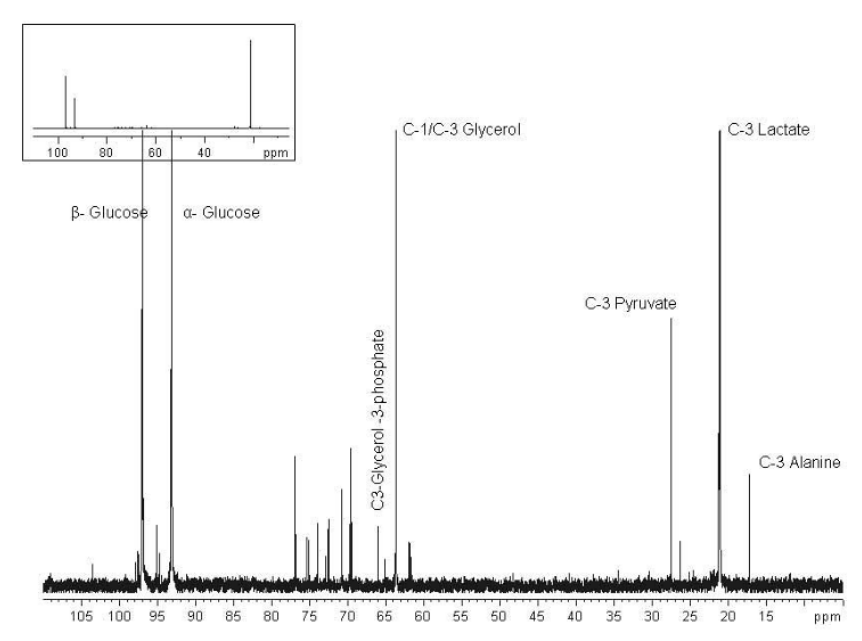

Figure I

Proton-decoupled ${ }^{13} \mathrm{C}-\mathrm{NMR}$ spectrum of erythrocyte-freed $P$. falciparum following incubation with $D$ [I-13C] glucose $(25 \mathrm{mM})$. Chemical shifts in parts per million were as follows: $\mathrm{C}$-I $\alpha$-glucose, 93.2; C-I $\beta$-glucose, 97.0; C-3 lactate, 21.2; C-I,3 glycerol, 63.6; C-3 pyruvate, 27.6; C-3 alanine, 17.3 and C-3 glycerol-3-phosphate, 65.9. Spectrum representative of a typical experiment $(n=4)$.

parasite. Glycerol has previously been postulated to be taken up by the malaria parasite from the host serum for the synthesis of lipids and membranes and recently the crystal structure of the aquaglycerolporin PfAQP, involved in the transport of glycerol, was solved [17]. However, data from this study suggest that glycerol can be generated by the malaria parasite during asexual growth. The formation of glycerol is unusual amongst eukaryotes (note glycerol is not produced by uninfected human erythrocytes [18], nor was it detected in control experiments), although it is a feature of anaerobic glucose catabolism in yeast [19], protozoan parasites such as trichomonas [20], leishmania [21] and trypanosomes [22], as well as rumen ciliates such as Dasytricha [23], Eudiplodinium [24], and Polyplastron [25]. Interestingly, in all of these studies ${ }^{13} \mathrm{C}$ NMR spectroscopy was the method adopted for the identification of glycerol. As glycerol is a poor chromophore and ionizes poorly in an ESI (electro-spray ionisation) source, complementary techniques such as HPLC or GCMS/MS and LC-MS/MS are either insensitive or non-selective. These problems can only be overcome with extensive derivatization of glycerol (e.g[26]). The relative analytical difficulties associated with detecting glycerol may explain why this metabolite has previously gone undetected in Plasmodium.

During anaerobic glycolysis, it is believed that glycerol is generated for the purposes of restoring the cell's redox balance. This occurs via an indirect mechanism known as the glycerol-3-phosphate shuttle that results in the oxidation of NADH and the transfer of electrons to the ETC. The shuttle consists of two components, cytosolic glycerol-3phosphate dehydrogenase and mitochondrial glycerol-3phosphate:ubiquinone oxidoreductase, both these components are present in $P$. falciparum [3]. At first sight, the need for such a shuttle to operate in $P$. falciparum may be questioned, as the production of lactate from glucose is redox neutral. However, as demonstrated from the study of yeast, growth (e.g. biomass production) is essentially a reductive process resulting in a net consumption of $\mathrm{NADPH}$ and a net production of NADH [19]. As a result cells have developed a number of mechanisms to re-oxidize NADH in various compartments. It would appear from data presented here that the malaria parasite possess a glycerol-3-phosphate shuttle to reoxidize cytosolic NADH whereas mitochondrial NADH may be re-oxidized using an alternative mechanism such as the type II NADH:ubiquinone oxidoreductase (alternative complex I) $[27,28]$.

At this stage it is uncertain how the malaria parasite generates glycerol. In yeast, glycerol is produced from the hydrolysis of the phosphate group of glycerol-3-phosphate via glycerol-3-phosphatase [29], however this enzyme appears to be missing from the genome of the human malaria parasite [3]. In trypanosomes, glycerol is generated from glycerol kinase operating in reverse with the concomitant formation of ATP [30-32]. In trypanosomes, this reaction occurs inside glycosomes where it is believed that the compartmentalisation of substrates at un-physiological concentrations (relative to the cytosol) allows this otherwise thermodynamically unfavourable reaction to take place $[30,31]$. Whether or not the $P$. falciparum glycerol kinase is similarly able to operate in reverse remains to be determined, however preliminary data would suggest that anaerobic incubation of $P$. falciparum parasites results in an increase in the intracellular levels of glycerol-3-phosphate (Biagini, unpublished).

\section{Conclusion}

This study shows that $P$. falciparum asexual parasites are able to generate glycerol from glucose. The production of glycerol is believed to be a metabolic adaptation to growth in $\mathrm{O}_{2}$-limited environments. These data support previous hypotheses that mitochondrial dehydrogenases such as PfNDH2 and G3PDH are important to the parasite for the purposes of redox balance under conditions of low $\mathrm{O}_{2}[28,33,34]$.

\section{Competing interests}

The authors declare that they have no competing interests.

\section{Authors' contributions}

L-YL carried out the NMR spectroscopy. MAH, MR, NF and $\mathrm{GAB}$ carried out the parasite culture, ${ }^{13} \mathrm{C}$-glucose incubations and sample preparations. PGB and SAW participated in the design of the study and drafting of the 
manuscript. GAB conceived the study, and participated in its design and coordination and prepared the manuscript. All authors read and approved the final manuscript.

\section{Acknowledgements}

This work was supported by grants from the Leverhulme Trust, the Wellcome Trust, the National Institute of Health Research (NHIR), the Northwest Development Agency (NWDA) and the Malaysian Government. The authors also thank the staff and patients of Ward 7Y and the Gastroenterology Unit, Royal Liverpool Hospital, for their generous donation of blood.

\section{References}

I. Bass CC, John FM: The cultivation of malaria plasmodia (Plasmodium vivax and Plasmodium falciparum)in vitro. J Exp Med 1912, 16:567-579.

2. Sherman IW: Biochemistry of Plasmodium (malarial parasites). Microbiol Rev 1979, 43:453-495.

3. Gardner MJ, Hall N, Fung E, White O, Berriman M, Hyman RW, Carlton JM, Pain A, Nelson KE, Bowman S, et al:: Genome sequence of the human malaria parasite Plasmodium falciparum. Nature 2002, 419:498-5II.

4. Vaidya $A B$, Mather MW: A post-genomic view of the mitochondrion in malaria parasites. Curr Top Microbiol Immunol 2005, 295:233-250.

5. van Dooren GG, Stimmler LM, McFadden GI: Metabolic maps and functions of the Plasmodium mitochondrion. FEMS Microbiol Rev 2006, 30(4):596-630.

6. Le Roch KG, Zhou Y, Blair PL, Grainger M, Moch JK, Haynes JD, De La Vega P, Holder AA, Batalov S, Carucci DJ, et al.: Discovery of gene function by expression profiling of the malaria parasite life cycle. Science 2003, 30 I: I 503-I508.

7. Bozdech Z, Llinas M, Pulliam BL, Wong ED, Zhu J, DeRisi JL: The transcriptome of the intraerythrocytic developmental cycle of Plasmodium falciparum. PLoS Biol 2003, I(I): E5.

8. Young JA, Fivelman QL, Blair PL, de la Vega P, Le Roch KG, Zhou Y, Carucci DJ, Baker DA, Winzeler EA: The Plasmodium falciparum sexual development transcriptome: a microarray analysis using ontology-based pattern identification. Mol Biochem Parasitol 2005, 143:67-79.

9. Daily JP, Scanfeld D, Pochet N, Le Roch K, Plouffe D, Kamal M, Sarr O, Mboup S, Ndir O, Wypij D, et al.: Distinct physiological states of Plasmodium falciparum in malaria-infected patients. Nature 2007, 450:955-956.

10. Trager $W$, Jensen JB: Human malaria parasites in continuous culture. Science 1976, 193:673-675.

II. Allen RJ, Kirk K: The membrane potential of the intraerythrocytic malaria parasite Plasmodium falciparum. J Biol Chem 2004, 279: I I 264- II 272

12. Saliba KJ, Kirk K: pH regulation in the intracellular malaria parasite, Plasmodium falciparum. $\mathbf{H}(+)$ extrusion via a v-type h(+)-atpase. J Biol Chem 1999, 274:33213-33219.

13. Biagini GA, Pasini EM, Hughes R, De Koning HP, Vial HJ, O'Neill PM, Ward SA, Bray PG: Characterization of the choline carrier of Plasmodium falciparum: a route for the selective delivery of novel antimalarial drugs. Blood 2004, 104:3372-3377.

14. Saliba KJ, Martin RE, Broer A, Henry RI, McCarthy CS, Downie MJ, Allen RJ, Mullin KA, McFadden GI, Broer S, et al:: Sodium-dependent uptake of inorganic phosphate by the intracellular malaria parasite. Nature 2006, 443:582-585.

15. Saliba KJ, Kirk K: H+-coupled pantothenate transport in the intracellular malaria parasite. J Biol Chem 200I, 276: $181 \mid 5-18121$.

16. London RE: ${ }^{13} \mathrm{C}$ labelling studies of metabolic regulation. Prog NuclMagnetic Resonance Spectroscopy 1988, 20:337-383.

17. Newby ZE, O'Connell J 3rd, Robles-Colmenares Y, Khademi S, Miercke LJ, Stroud RM: Crystal structure of the aquaglyceroporin PfAQP from the malarial parasite Plasmodium falciparum. Nat Struct Mol Biol 2008, 15(6):619-625.

18. Brown FF, Campbell ID: N.m.r. studies of red cells. Philos Trans $R$ Soc Lond B Biol Sci 1980, 289:395-406.

19. Bakker BM, Overkamp KM, van Maris AJ, Kotter P, Luttik MA, van Dijken JP, Pronk JT: Stoichiometry and compartmentation of
NADH metabolism in Saccharomyces cerevisiae. FEMS Microbiol Rev 200I, 25: 15-37.

20. Chapman A, Linstead DJ, Lloyd D, Williams J: I3C-NMR reveals glycerol as an unexpected major metabolite of the protozoan parasite Trichomonas vaginalis. FEBS Lett 1985, | $91: 287-292$.

21. Darling TN, Davis DG, London RE, Blum J]: Products of Leishmania braziliensis glucose catabolism: release of D-lactate and, under anaerobic conditions, glycerol. Proc Natl Acad Sci USA 1987, 84:7|29-7|33.

22. Mackenzie NE, Hall JE, Flynn IW, Scott Al: I3C nuclear magnetic resonance studies of anaerobic glycolysis in Trypanosoma brucei spp. Biosci Rep 1983, 3:141-I51.

23. Ellis JE, Mclntyre PS, Saleh M, Williams AG, Lloyd D: Influence of $\mathrm{CO} 2$ and low concentrations of $\mathrm{O} 2$ on fermentative metabolism of the rumen ciliate Dasytricha ruminantium. J Gen Microbiol | 99|, |37:|409-|4|7.

24. Ellis JEMPS, Saleh M, Williams AG, Lloyd D: Influence of ruminal concentrations of $\mathrm{O} 2$ and $\mathrm{CO} 2$ on fermentative metabolism of the rumen entodiniomorphid ciliate Eudiplodinium maggii. Curr Microbiol I99I, 23:245-25I.

25. Ellis JE, Mclntyre PS, Saleh M, Williams AG, Lloyd D: Influence of $\mathrm{CO} 2$ and low concentrations of $\mathrm{O2}$ on fermentative metabolism of the ruminal ciliate Polyplastron multivesiculatum. Appl Environ Microbiol I99I, 57:1400- 4007.

26. McIntosh TS, Davis HM, Matthews DE: A liquid chromatographymass spectrometry method to measure stable isotopic tracer enrichments of glycerol and glucose in human serum. Anal Biochem 2002, 300:163-169.

27. Biagini GA, Viriyavejakul P, O'Neill PM, Bray PG, Ward SA: Functional characterization and target validation of alternative complex I of Plasmodium falciparum mitochondria. Antimicrob Agents Chemother 2006, 50(5):|84|-|85|.

28. Fisher N, Bray PG, Ward SA, Biagini GA: The malaria parasite type II NADH:quinone oxidoreductase: an alternative enzyme for an alternative lifestyle. Trends Parasitol 2007, 23:305-310.

29. Norbeck J, Pahlman AK, Akhtar N, Blomberg A, Adler L: Purification and characterization of two isoenzymes of DL-glycerol3-phosphatase from Saccharomyces cerevisiae. Identification of the corresponding GPP I and GPP2 genes and evidence for osmotic regulation of Gpp2p expression by the osmosensing mitogen-activated protein kinase signal transduction pathway. J Biol Chem 1996, 27 I: I 3875-I388I.

30. Hammond DJ, Bowman IB: Trypanosoma brucei: the effect of glycerol on the anaerobic metabolism of glucose. Mol Biochem Parasitol 1980, 2:63-75.

31. Hammond DJ, Bowman IB: Studies on glycerol kinase and its role in ATP synthesis in Trypanosoma brucei. Mol Biochem Parasitol 1980, 2:77-91.

32. Hellemond JJ, Bakker BM, Tielens AG: Energy metabolism and its compartmentation in Trypanosoma brucei. Adv Microb Physiol 2005, 50:199-226.

33. Fisher N, Bray PG, Ward SA, Biagini GA: Malaria-parasite mitochondrial dehydrogenases as drug targets: too early to write the obituary. Trends Parasitol 2008, 24:9-10.

34. Painter HJ, Morrisey JM, Mather MW, Vaidya AB: Specific role of mitochondrial electron transport in blood-stage Plasmodium falciparum. Nature 2007, 446:88-91. 